\title{
Shikonin potentiates the effect of arsenic trioxide against human hepatocellular carcinoma in vitro and in vivo
}

\author{
Jingjing Song ${ }^{1, *}$, Zhongwei Zhao ${ }^{1, *}$, Xiaoxi Fan $^{1, *}{ }^{*}$ Minjiang Chen ${ }^{1}$, Xingyao Cheng ${ }^{2}$, \\ Dengke Zhang ${ }^{1}$, Fazong $\mathbf{W u}^{1}$, Xihui Ying ${ }^{1}$, Jiansong $\mathrm{Ji}^{1}$ \\ ${ }^{1}$ Department of Interventional Radiology, The Fifth Affiliated Hospital of Wenzhou Medical University, Zhejiang University \\ Lishui Hospital, Lishui, Zhejiang 323000, China \\ ${ }^{2}$ Department of Radiology, The Fifth Affiliated Hospital of Wenzhou Medical University, Zhejiang University Lishui Hospital, \\ Lishui, Zhejiang 323000, China \\ *These authors have contributed equally to this work \\ Correspondence to: Jiansong Ji, email: jjstcty@sina.com \\ Jingjing Song, email: 8808s_j996@163.com
}

Keywords: arsenic trioxide, shikonin, ROS, endoplasmic reticulum stress, hepatocellular carcinoma

Received: May 27, $2016 \quad$ Accepted: September 02, 2016

Published: September 15, 2016

\section{ABSTRACT}

Hepatocellular carcinoma (HCC) is a highly lethal malignancy mostly because of metastasis, recurrence and acquired resistance to conventional chemotherapy. Arsenic trioxide (ATO) is successfully used to treat hematological malignancies, and has been proven to trigger apoptosis in HCC cells. However, the phase II trial evaluating the efficacy and toxicity of ATO in patients with HCC showed that singleagent ATO is poorly active against HCC. Therefore, it is of great importance to develop effective chemosensitization agents to ATO. The aim of the present study was to determine whether shikonin (SHI), a natural product from the root of lithospermum erythrorhizon, could synergistically enhance the anti-HCC efficacy of ATO both in vitro and in vivo. We found that the combination of SHI and ATO exhibited synergistic anticancer efficacy and achieved greater selectivity between cancer cells and normal cells. By inducing intracellular oxidative stress, SHI potentiated ATO-induced DNA damage, followed by increased activation of endoplasmic reticulum stress. In addition, inhibition of ROS reversed the apoptosis induced by SHI and ATO, and recovered the activation of endoplasmic reticulum stress, which revealed the vital role of ROS in the synergism. Moreover, HepG2 xenograft tumor growth in nude mice was more effectively inhibited by combined treatment with SHI and ATO. These data suggest that the combination of SHI with ATO presents a promising therapeutic approach for the treatment of HCC.

\section{INTRODUCTION}

Hepatocellular carcinoma (HCC) is the most common liver malignancy worldwide, and its incidence is increasing [1]. Despite the ongoing improvement in management of HCC, little success has been achieved in ameliorating the situation of this disease [2]. Currently, there are few effective therapies partly because the molecular- and cell-based mechanisms that contribute to the pathogenesis of this cancer type are little understood. Systemic chemotherapy is one of the few treatment choices for those unresectable HCC patients [3]. Sorafenib, an oral multikinase inhibitor, is the current standard for the treatment of unresectable HCC [4]. However, the clinical efficacy of sorafenib is modest, and some patients are intolerant [5]. Thus, more effective therapeutic strategies for advanced HCC are needed.

Arsenic trioxide (ATO) is an effective anticancer drug used in patients with relapsed acute promyelocytic leukemia (APL), and is intensively investigated for treatment of other tumors [6,7]. However, ATO is much less effective against solid tumors such as HCC, colon carcinoma and gastric cancer. Moreover, chronic exposure to low-concentrations of ATO $(1 \mathrm{mg} / \mathrm{kg})$ can enhance tumor growth and metastasis, but cannot induce tumor cell death in a mouse model of HCC [8]. The phase II study evaluating the clinical and biologic effects of ATO in patients with $\mathrm{HCC}$ indicated that ATO is poorly effective 
[9]. Recently, intensive combination chemotherapy has been considered for the treatment of cancer, and found that combination chemotherapy had a better effects than those treated with single-agent therapy $[10,11]$. Hence, searching of effective agents that could increase the effect of ATO and reduce side effects is very important.

Reactive oxygen species (ROS) are chemically reactive molecules that have important functions in mammalian cells. Mounting research reveals that, compared with their normal counterparts, cancer cells have increased levels of ROS and are under oxidative stress due to an imbalanced redox status $[12,13]$. Consequently, cancer cells are more sensitive to agents that further increase ROS levels and oxidative stress [14, 15]. Recent studies have revealed that ATO-induced apoptosis in cancer cells is partially mediated through ROS production $[16,17]$. Therefore, chemical agents which can alter the antioxidant defenses and induce oxidative stress have been proposed as potential candidates for enhancing the efficacy of ATO in cancer treatment.

Shikonin (SHI), a natural product isolated from the lithospermum erythrorhizon, has been identified as a multifunctional bioactive natural product. Numerous studies have shown that SHI induces apoptosis in cancer cells in vitro and in vivo [18, 19]. These anti-tumor activities are attributed to the regulation of cell cycle, apoptosis and invasion of tumor cells. A recent study showed that SHI is able to induce apoptosis in human promyelocytic leukemia HL-60 cells through generation of ROS [20]. SHI treatment significantly induced ROS production in cancer cells but not in normal cells, which may underlie SHI's selective cancer killing ability [20, 21]. Moreover, shikonin is able to induce apoptosis in HCC cells via increasing ROS production [22]. In the present study, we intended to identify the hypothesis that SHI, a natural inducer of ROS, could enhance the cytotoxicity of ATO in HCC cells. In addition, we investigated the possible mechanisms underlying cell death induced by combined treatment with SHI and ATO in HCC cells.

\section{RESULTS}

\section{SHI potentiates the proliferation inhibition effect of ATO in HCC cells in vitro}

We first determined the effect of combination therapy of SHI and ATO on viability of HCC cells. As show in Figure 1A, SHI treatment alone did not significantly decreased the viability of HepG2 cells. However, combination of SHI and ATO exhibited better cytotoxicity on HepG2, Hep3B and Huh7 cells than the use of a single agent (Figure 1A-1C). Moreover, combination of SHI and ATO displayed less cytotoxicity toward HL-7702 normal human liver cells, suggesting the selectivity of SHI in combination ATO (Figure 1D). These results suggested that SHI can sensitize HCC cell lines to the cell killing effects of ATO.

\section{SHI potentiates the apoptotic effects of ATO in HCC cells}

To determine whether the growth inhibition of HCC cells by combined treatment was caused by apoptosis, we then evaluated the pro-apoptotic effect of SHI, ATO and ATO+SHI on HepG2 and Hep3B cells. As shown in Figure $2 \mathrm{~A}-2 \mathrm{D}$, combined treatment with SHI and ATO resulted in a significant increase in the number of apoptotic cells compared with SHI or ATO alone. We confirmed these findings by caspase acitivity assay and found that SHI markedly increased ATO-induced caspase 3 and caspase 9 activation, suggesting the activation of apoptosis pathway (Figure 2E-2F). The levels of apoptosis-related proteins were also examined by western blotting analysis. As shown in Figure 2G-2J, SHI and ATO cotreatment markedly reduced the levels of pro-caspase 3 and anti-apoptotic protein Bcl-2, and increased the levels of cle-caspase 3 and Bax. Taken together, our findings indicated that combined treatment reduced HCC cells growth through induction of apoptosis.

\section{ER stress was involved in the SHI and ATO combined treatment-induced apoptosis in vitro}

Studies have shown that ER stress has been implicated in ATO-induced apoptosis [23, 24]. Besides, SHI could selectively kill cancer cells by activating ER stress [25]. Therefore, we suspected that activation of ER stress contributes to HCC cell apoptosis by combined treatment. We examined the expression of ER stressrelated proteins including eukaryotic initiation factor $2 \alpha$ (p-EIF2 $\alpha$ ) and activating transcription factor-4 (ATF4) in combined treated HCC cells. As shown in Figure $3 \mathrm{~A}-3 \mathrm{~B}$, treatment of cells with SHI $(1 \mu \mathrm{M})$ and ATO $(6$ $\mu \mathrm{M})$ alone both slightly increased the levels of p-EIF $2 \alpha$ and ATF4, but combined treatment with SHI and ATO significantly increased the levels of p-EIF $2 \alpha$ and ATF4. Meanwhile, we found that combined treatment resulted in significant increases in the mRNA and protein levels of CHOP (Figure 3C-3D). We further investigated whether ER stress was involved in the anti-tumor effects of combined treatment. As shown in Figure 3E-3F, 4-PBA pre-treatment reduced combined treatment-induced CHOP mRNA and protein levels. Meanwhile, we found that 4-PBA pre-treatment markedly decreased combined treatment-induced apoptosis in HepG2 cells (Figure 3G). These results suggested that ER stress was involved in combined treatment-induced HCC cell apoptosis.

\section{SHI potentiates ATO-induced apoptosis by ROS accumulation}

ROS promote cell survival and growth by regulating cell signaling cascades [26, 27]. However, over production of ROS may lead to oxidative stress and cell apoptosis [28, 29]. SHI has been found to induce the accumulation of 
ROS and oxidative stress in cancer cells [20, 30]. Based on these findings, we investigated whether intracellular ROS generation was involved in the anti-tumor effects of combined treatment. As shown in Figure 4A, treatment of cells with SHI $(1 \mu \mathrm{M})$ and ATO $(6 \mu \mathrm{M})$ alone both slightly increased intracellular ROS levels as detected by increased DCF levels. However, treatment of HepG2 cells with SHI and ATO significantly increased intracellular ROS levels. This increase was evident as early as 20 min following exposure to SHI and ATO and remained elevated for 2 hours. To determine whether combined treatment-induced ROS participates in mediating cell apoptosis, we utilized $\mathrm{N}$-acetyl cysteine (NAC) and L-glutathione reduced (GSH). Our results showed that pre-treatment of HepG2 cells with NAC and GSH significantly reduced the inhibition of cell proliferation induced by combined treatment (Figure 4B). Furthermore, NAC markedly reversed combined treatmentinduced apoptosis in HepG2 and Hep3B cells (Figure 4C4F). Meanwhile, the activation of caspase 3 and caspase 9 were also reversed (Figure 4G-4H).
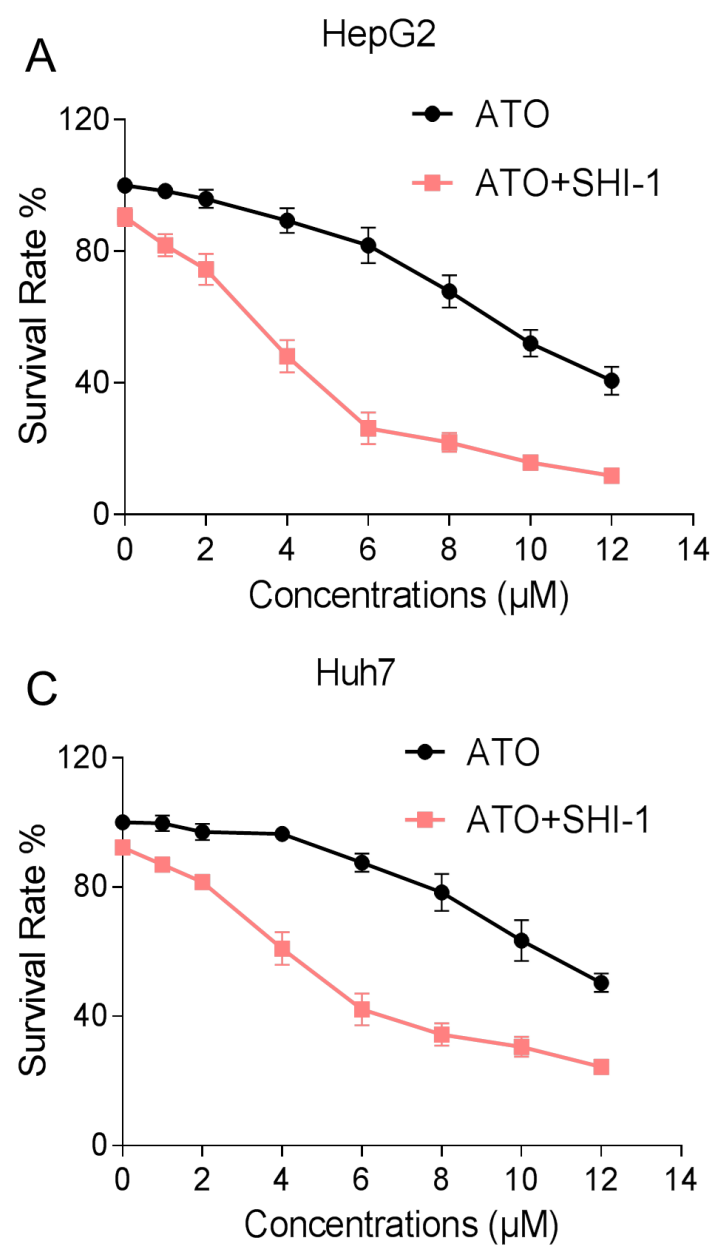

Furthermore, we detected the reverse effect of NAC on combined treatment-induced ER stress in HepG2 cells. As shown in Figure 5A-5B, pre-treatment with NAC markedly reversed the overexpression of $\mathrm{p}$-EIF $2 \alpha$ and ATF4 in combined treatment-treated HepG2 cells. In addition, NAC pre-treatment significantly reversed combined treatment-induced increase in CHOP mRNA and protein levels (Figure 5C-5D). Meanwhile, the increase of Bax and the reduction of $\mathrm{Bcl}-2$ induced by combined treatment were both blocked by NAC pretreatment (Figure 5E-5F). These findings indicated that ROS might be the upstream mediator for SHI to increase the apoptosis-inducing ability of ATO in HCC cells.

\section{SHI increases the anticancer activity of ATO against HCC cells in vivo}

Our last objective was to confirm our in vitro findings in a HCC cancer xenograft model. We injected HepG2 cells in athymic nu/nu mice. When the tumors
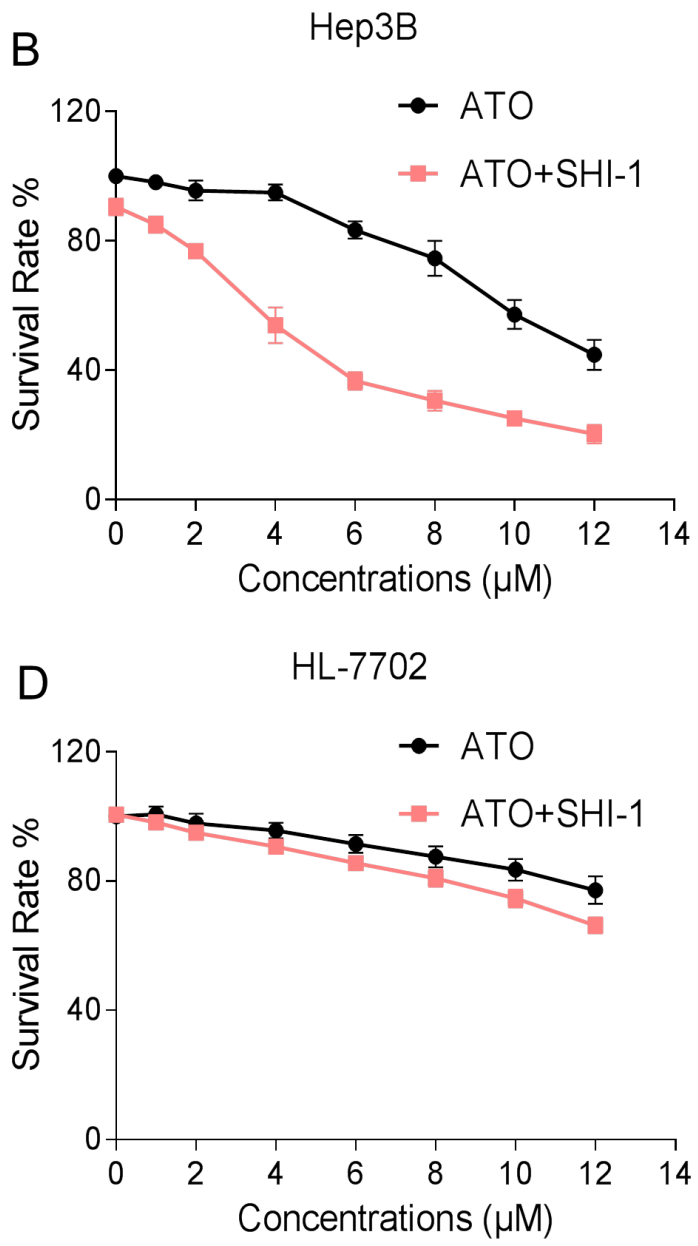

Figure 1: SHI potentiates the proliferation inhibition effect of ATO in HCC cells in vitro. A-C. SHI enhances ATO-induced growth inhibition in HCC cells. Briefly, cells were treated with SHI and ATO for $24 \mathrm{~h}$, cell viability was determined by MTT assay. Assays were performed in triplicate. D. SHI and ATO combined treatment showed less cytotoxicity toward HL-7702 normal human liver cells. HL-7702 cells were treated with SHI and ATO for 24 h, cell viability was determined by MTT assay. Assays were performed in triplicate. 
A

$\bar{n}$

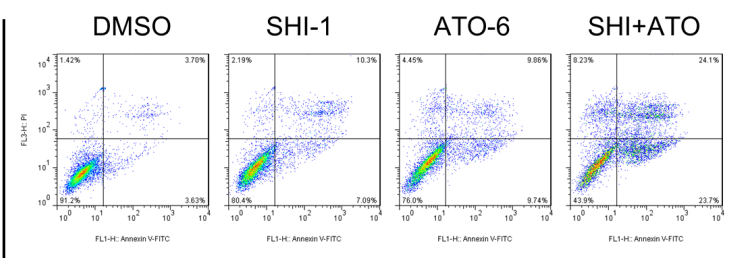

Annexin V

C

$\bar{\alpha}$

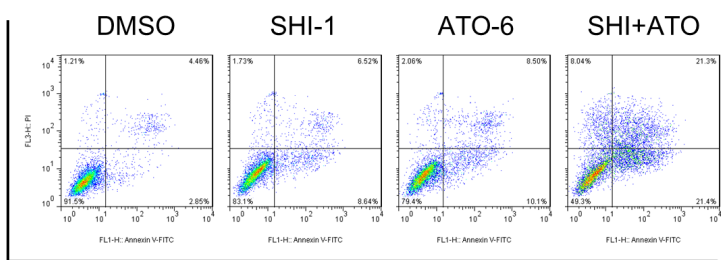

Annexin V

E

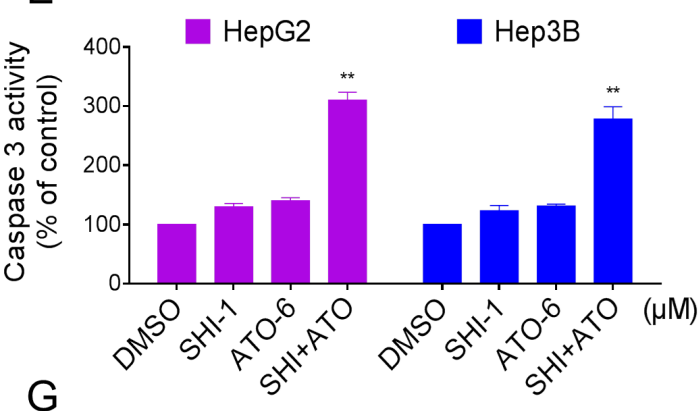

ATO $(6 \mu \mathrm{M})$

SHI $(1 \mu \mathrm{M})$

Pro-Caspase 3

Cle-Caspase 3

GAPDH

$\mathrm{H}$

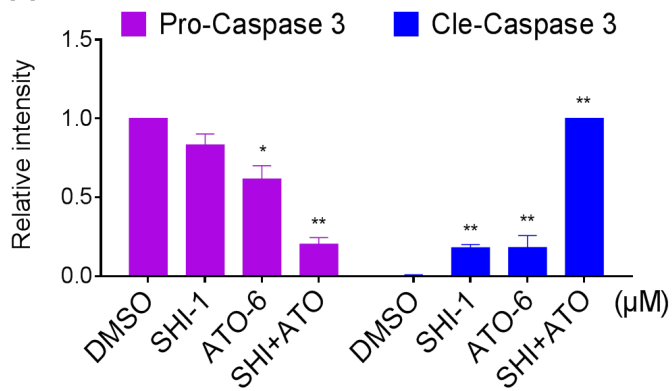

F

$\mathrm{J}$
B

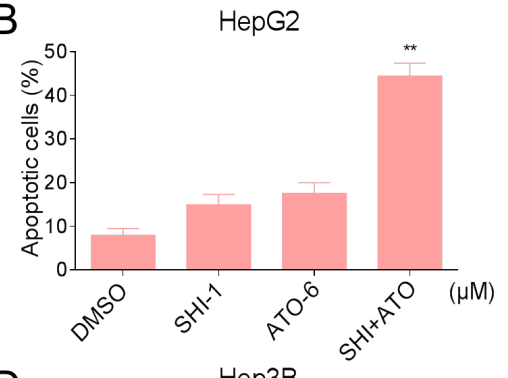

D

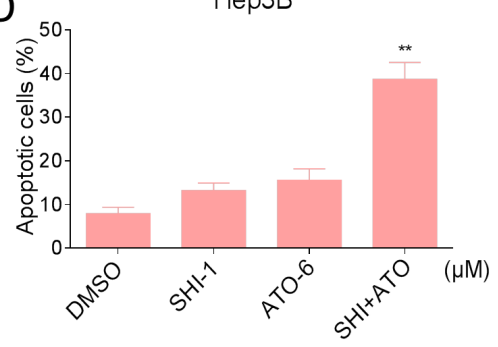

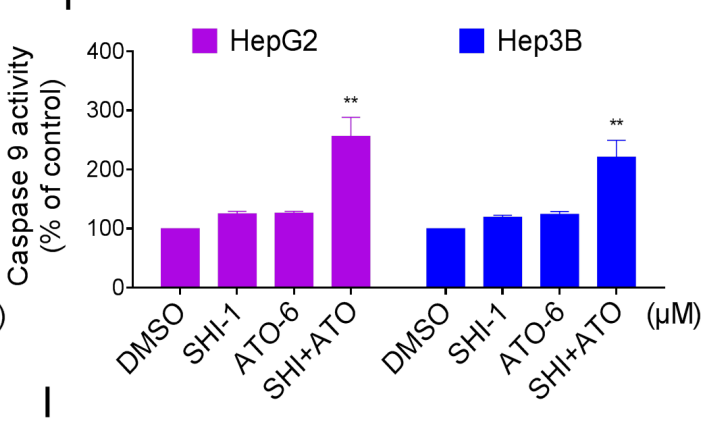

ATO $(6 \mu \mathrm{M})$

$\mathrm{SHI}(1 \mu \mathrm{M})$

Bcl-2

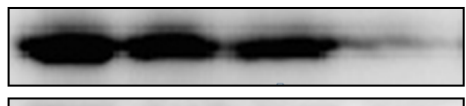

Bax

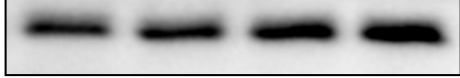

GAPDH
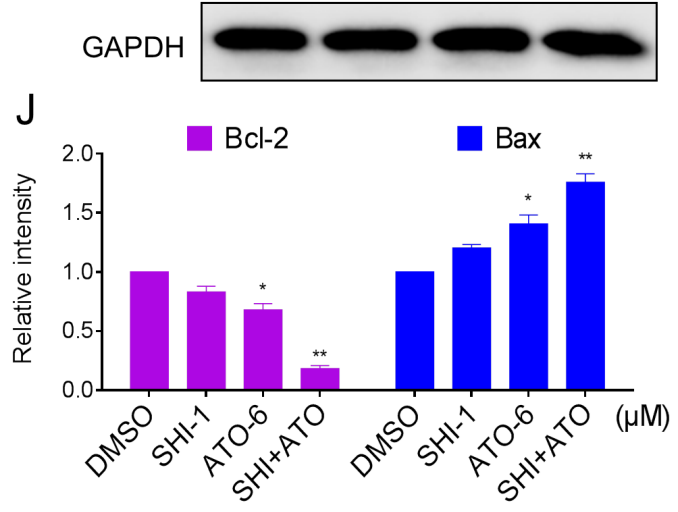

Figure 2: SHI potentiates the apoptosis-inducing effect of ATO in HCC cells in vitro. A. SHI enhances ATO-induced apoptosis in HepG2 cells. Cells after treatment for $24 \mathrm{~h}$ were collected and stained with Annexin V and PI. Cell apoptosis rate was analyzed by flow cytometer. Similar results were obtained in three independent experiments. B. The percentage of apoptotic cells in the treatment groups was calculated. C. SHI enhances ATO-induced apoptosis in Huh7 cells. D. The percentage of apoptotic cells in the treatment groups was calculated. E-F. Cells after treatment for $20 \mathrm{~h}$ were lysed and cell proteins were used to determine caspase 3 and caspase 9 activities with a fluorescence assay kit using specific substrates. G-J. Western blot analysis of expression levels of caspases, Bcl-2 and Bax in HepG2 cells. Cells after treatment for $20 \mathrm{~h}$ were collected and lysed, cell proteins $(50 \mu \mathrm{g})$ were separated by SDS-PAGE and immunoblotted with specific primary antibodies. All images shown here are representative of three independent experiments with similar results. $* * \mathrm{P}<0.01$ versus control group. 
A

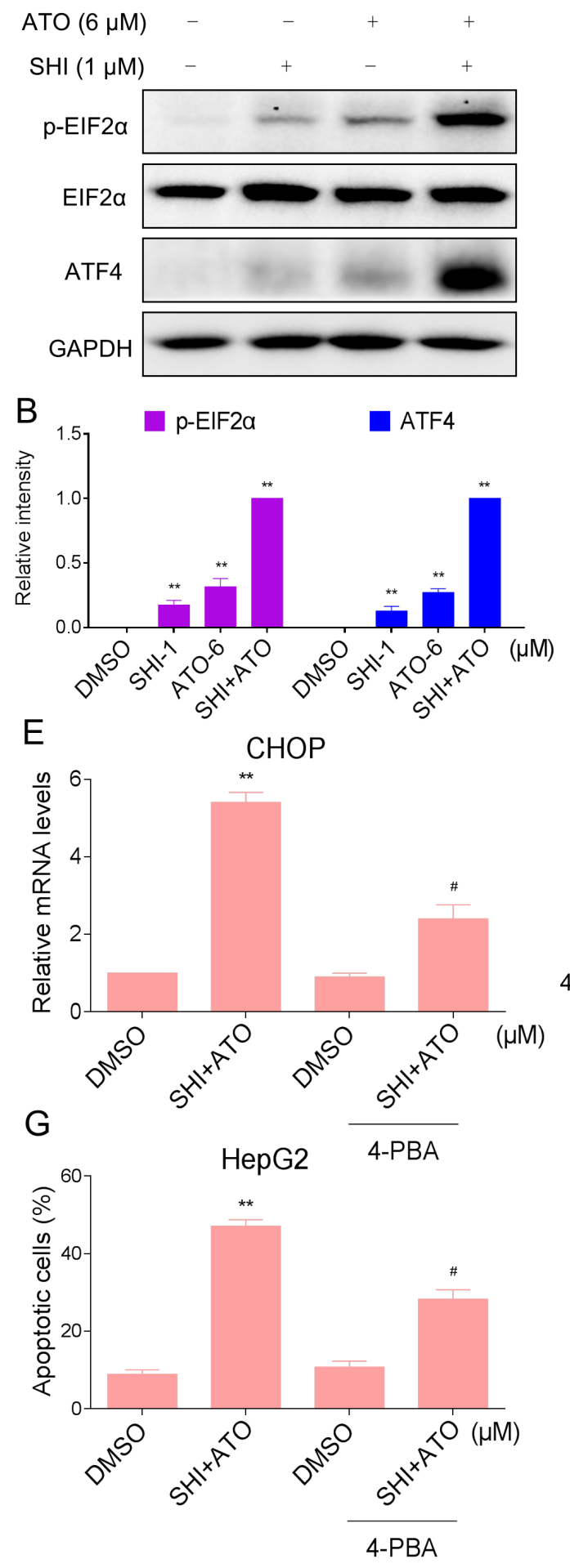

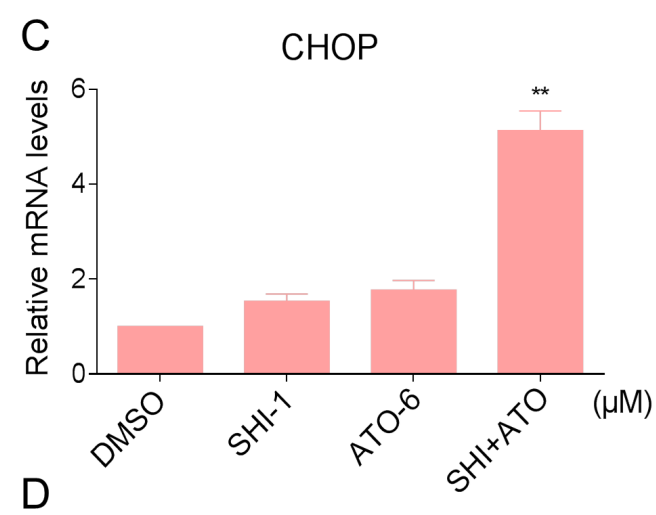

$\begin{array}{lllll}\operatorname{ATO}(6 \mu \mathrm{M}) & - & - & + & + \\ \operatorname{SHI}(1 \mu \mathrm{M}) & - & + & - & +\end{array}$
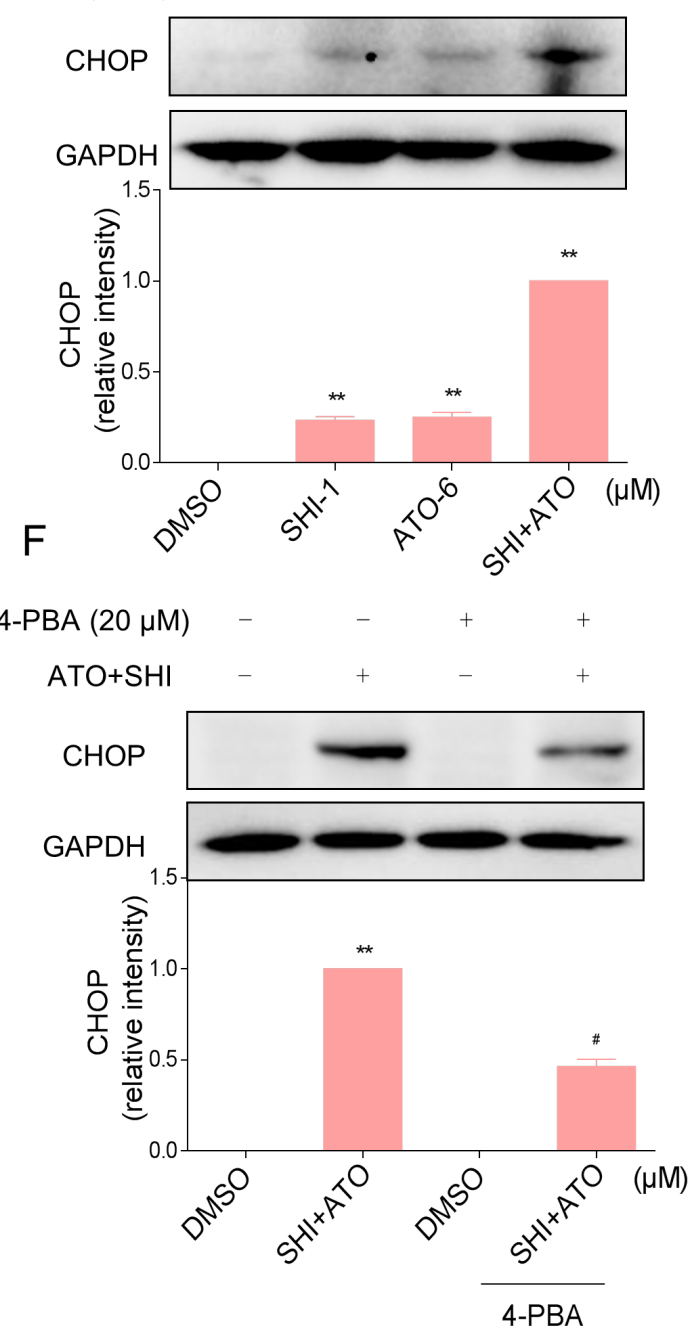

Figure 3: ER stress was involved in the SHI and ATO combined treatment-induced apoptosis in vitro. A-B. Combined treatment increased the expression of p-EIF2 $\alpha$ and ATF4 proteins. HepG2 cells were incubated with SHI or/and ATO for $6 \mathrm{~h}$ and then the protein levels of p-EIF2 $\alpha$ and ATF4 were analyzed by western blotting. A set of representative results is shown from three independent experiments. C. Combined treatment increased CHOP mRNA levels in HepG2 cells. HepG2 cells were treated with SHI or/and ATO for $6 \mathrm{~h}$. The mRNA expression of CHOP was analyzed by qRT-PCR. D. Combined treatment increased CHOP protein levels in HepG2 cells. HepG2 cells were incubated with SHI or/and ATO for $12 \mathrm{~h}$ and then the protein levels of CHOP was analyzed by western blotting. E-G. 4-PBA could partially attenuated combined treatment-induced HepG2 cells apoptosis. (E) The mRNA expression of CHOP was analyzed by qRT-PCR. (F) The protein expression of CHOP was analyzed by western blotting. (G) Cell apoptosis rate was analyzed by flow cytometer. ${ }^{* *} \mathrm{P}<0.01$ versus control group, ${ }^{*} \mathrm{P}<0.05$ versus combined treatment group. 
A

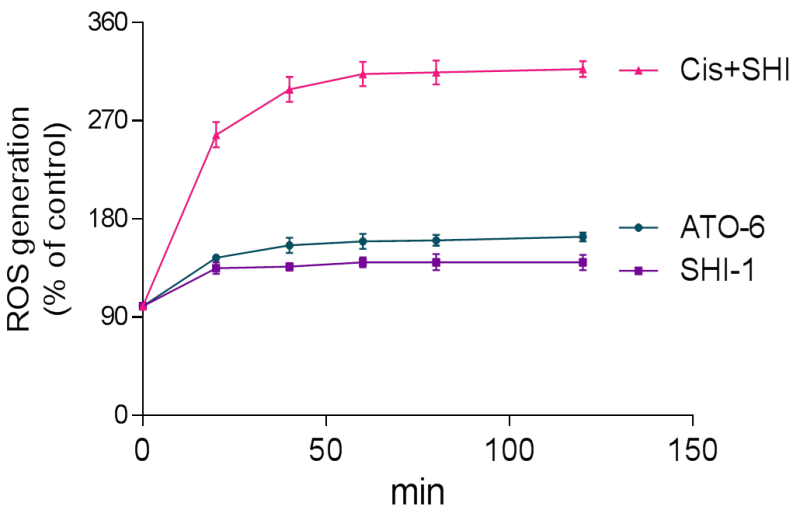

C

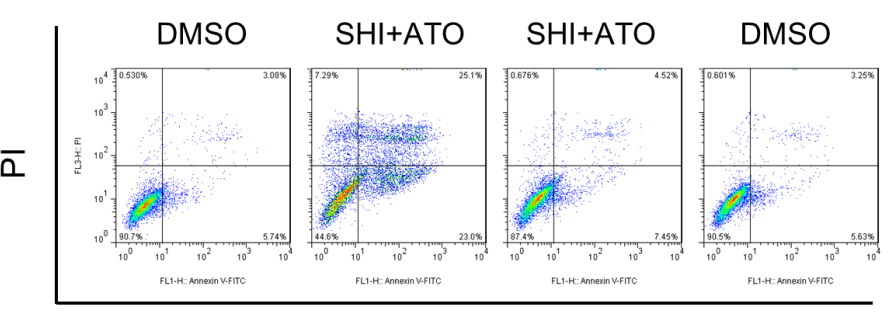

Annexin V

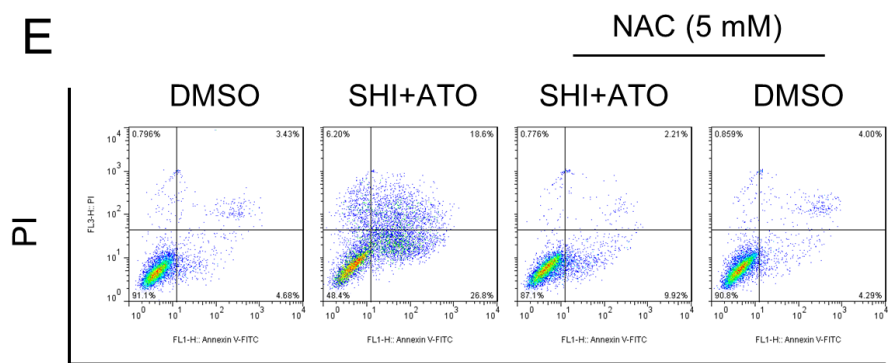

Annexin V

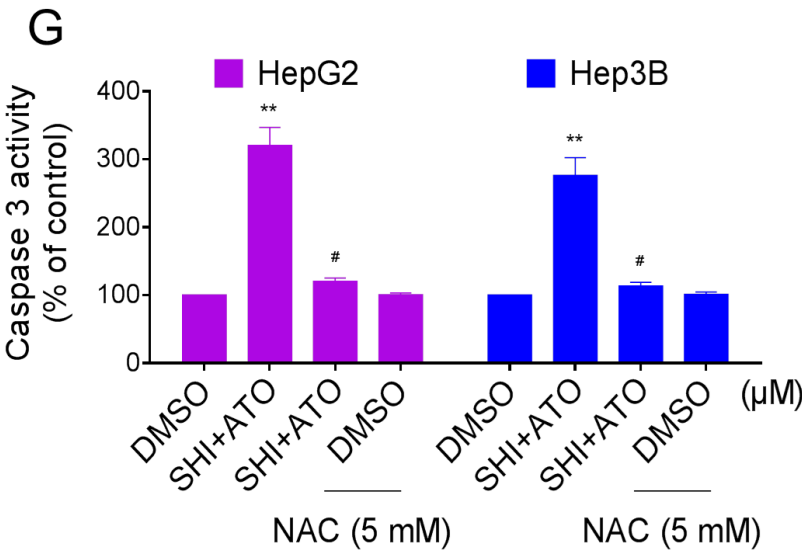

B

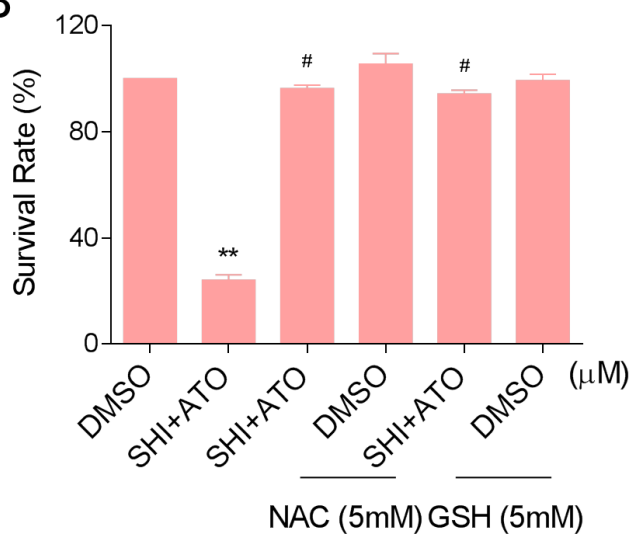

D

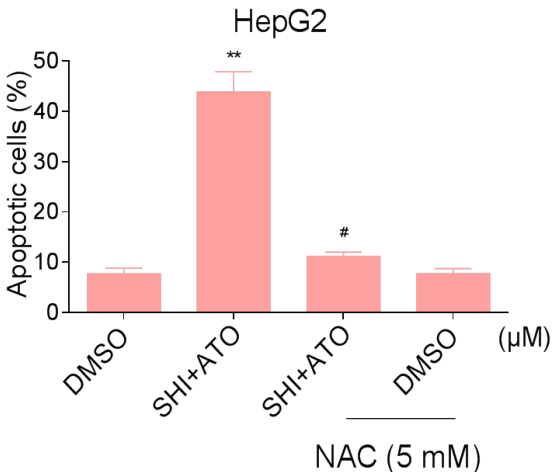

F Hep3B

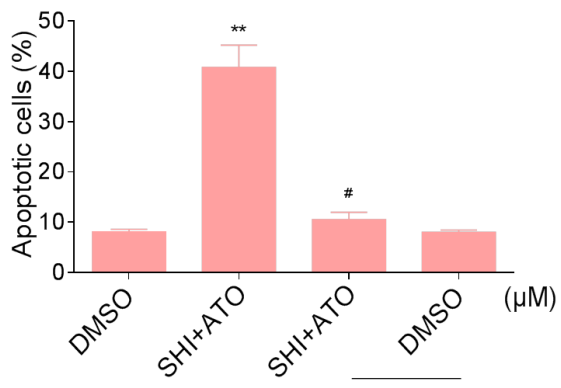

NAC (5 mM)

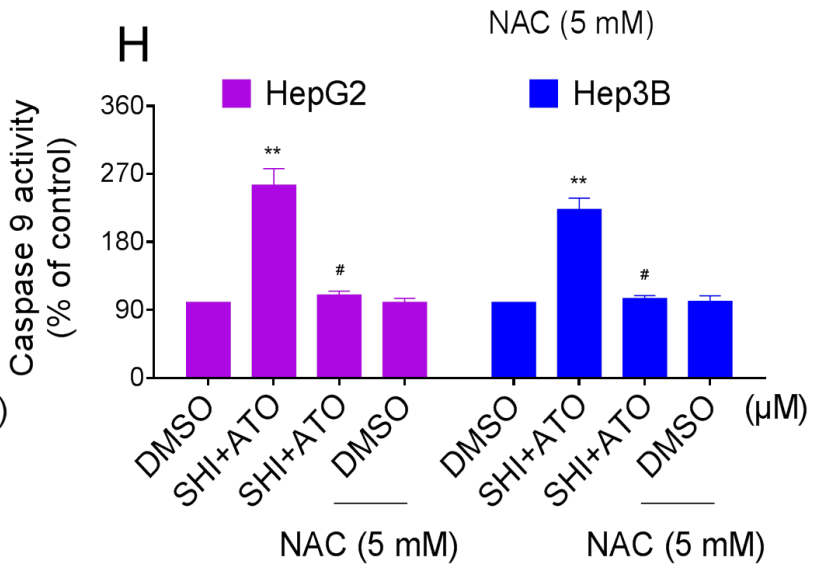

Figure 4: SHI potentiates ATO-induced apoptosis by ROS Accumulation. A. Intracellular ROS generation induced by SHI and/or ATO was measured by staining with DCFH-DA $(10 \mu \mathrm{M})$ and flow cytometer. B. HepG2 cells were pre-incubated with or without 5 mM NAC or GSH for $2 \mathrm{~h}$ before combined treatment. Cell viability was determined by MTT assay. C-F. NAC addition protects HCC cells from combined treatment-induced apoptosis. Percentage of cell apoptosis was determined by Annexin-V/PI staining and flow cytometer. G-H. NAC addition reverses combined treatment-induced activation of caspase 3 and caspase 9 . ${ }^{* *} \mathrm{P}<0.01$ versus control group, ${ }^{*} \mathrm{P}<0.05$ versus combined treatment group. 
A
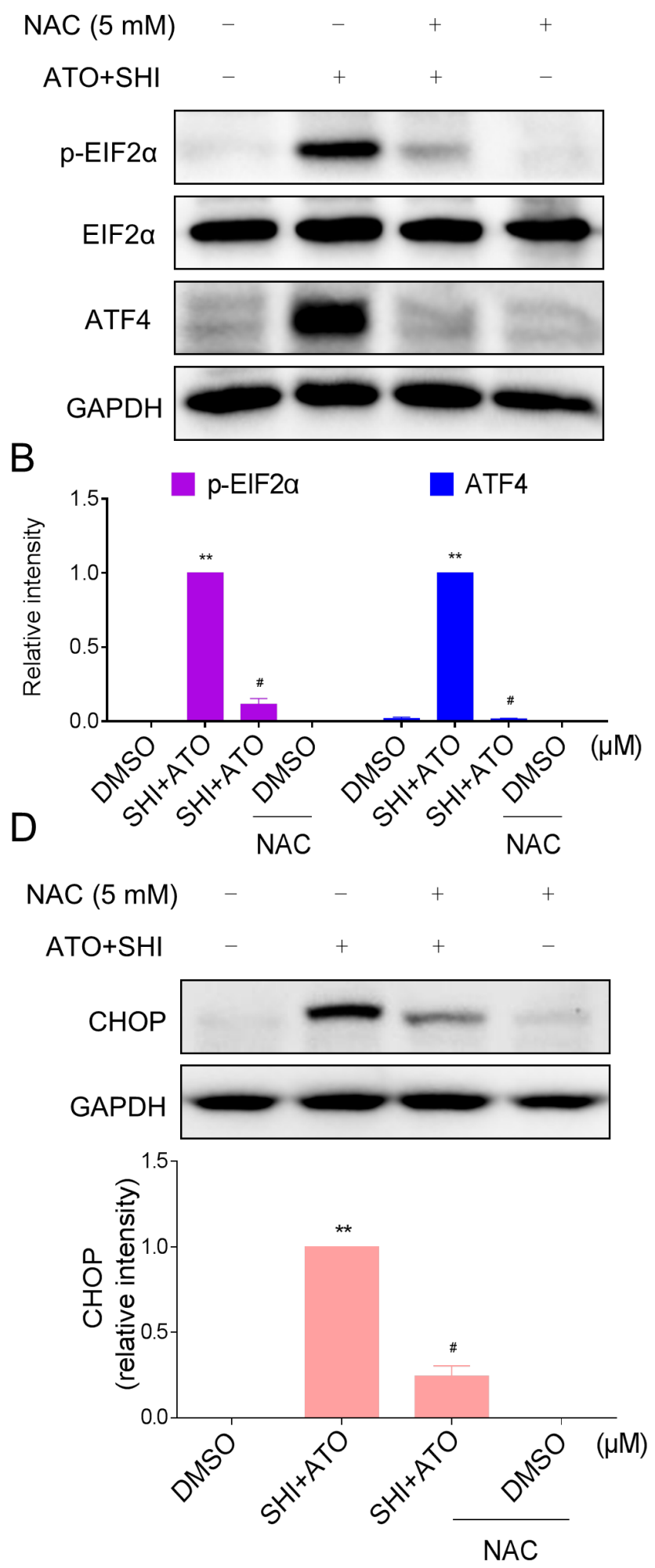

C

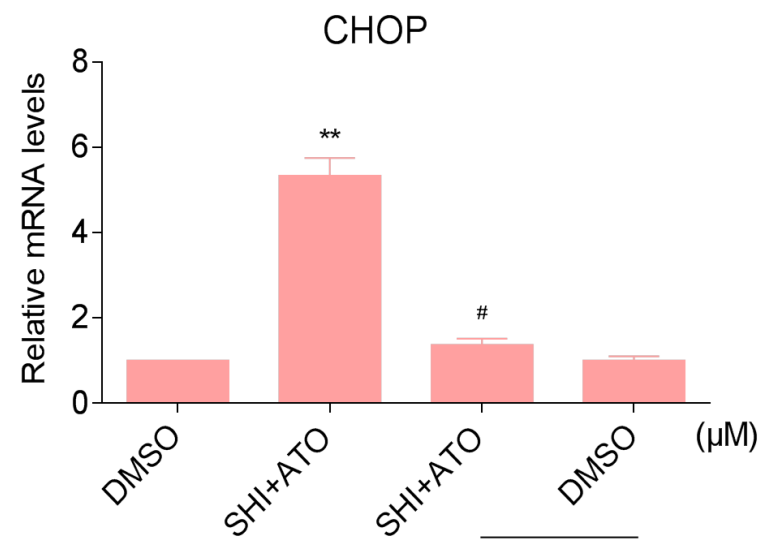

E

$\mathrm{NAC}(5 \mathrm{mM})$

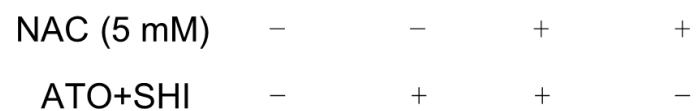

$\mathrm{F}$

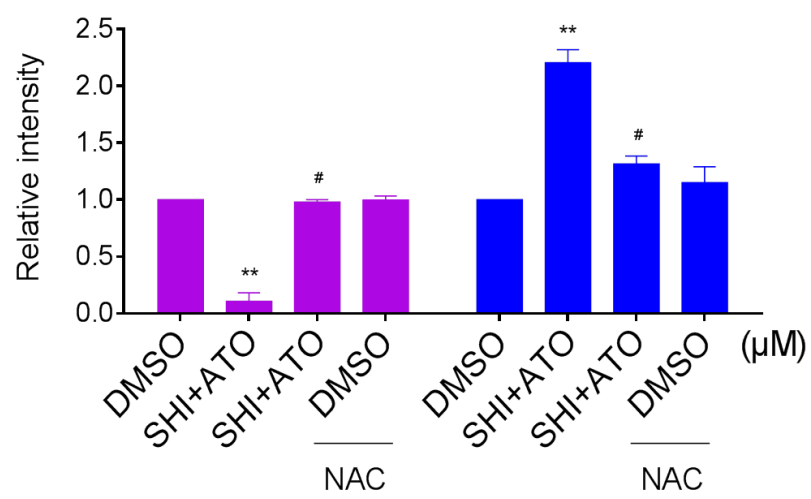

Figure 5: Blocking of ROS generation reversed the activation of ER stress induced by combined treatment. A-B. HepG2 cells were pre-incubated with or without $5 \mathrm{mM} \mathrm{NAC}$ for $2 \mathrm{~h}$ before combined treatment. Cell lysates were subjected to western blotting to analyze the expression of p-EIF2 $\alpha$ and ATF4. C. HepG2 cells were pre-incubated with or without $5 \mathrm{mM} \mathrm{NAC}$ for $2 \mathrm{~h}$ before combined treatment. The mRNA expression of CHOP was analyzed by qRT-PCR. D. HepG2 cells were pre-incubated with or without $5 \mathrm{mM}$ NAC for $2 \mathrm{~h}$ before combined treatment. The protein expression of CHOP was analyzed by western blotting. E-F. The protein levels of Bcl-2 and Bax were analyzed by western blotting. ${ }^{* *} \mathrm{P}<0.01$ versus control group, ${ }^{*} \mathrm{P}<0.05$ versus combined treatment group. 
grew to about $100 \mathrm{~mm}^{3}$, mice were treated with indicated compounds. As shown in Figure 6A, treatment of ATO alone inhibited HCC cancer cell growth in mice. However, combined treatment with SHI and ATO markedly reduced HepG2 tumor volume and weight compared to the vehicle-treated group. Importantly, there was no significant difference in body weight among the vehicle and combined-treated groups (Figure 6A-6C). Mechanistically, we found that combined treatment showed stronger ability in activating caspases activities in tumor tissues (Figure 6D). Besides, combined treatment increased the expression of CHOP mRNA, indicating that combined treatment-induced apoptosis in HepG2 cells is associated with activation of ER- stress in vivo (Figure 6E). Moreover, our results showed that combined treatment with SHI and ATO markedly increased the levels of MDA (Figure 6F). These findings indicated that that SHI can synergistically enhance ATOinduced tumor growth inhibition in vivo by inducing ROS accumulation.

\section{DISCUSSION}

Hepatocellular carcinoma (HCC) continues to be a global health problem. Sorafenib, an oral multikinase inhibitor with antiproliferative and antiangiogenic effects, currently sets the new standard for unresectable HCC. However, the objective tumor response rates are
A

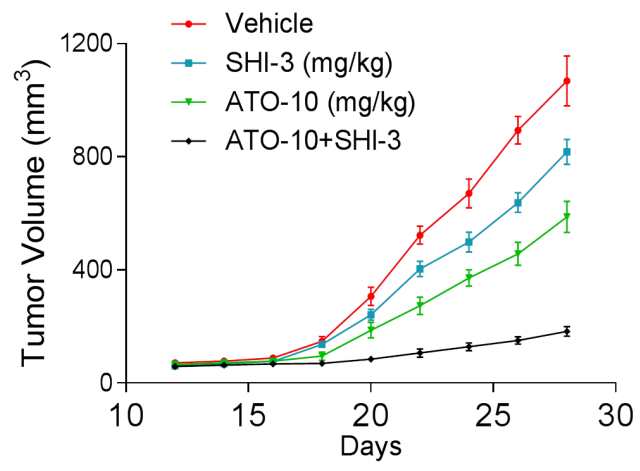

C

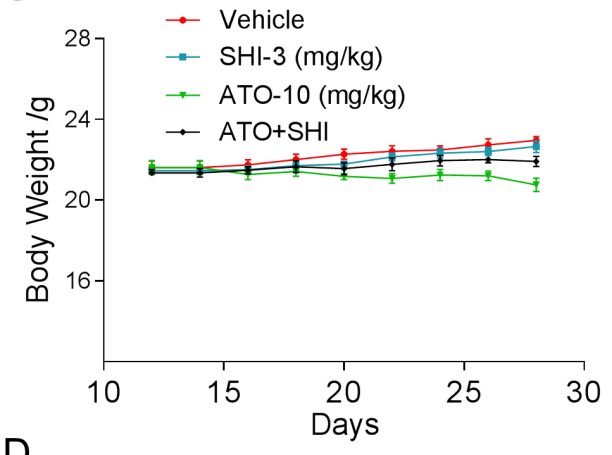

D

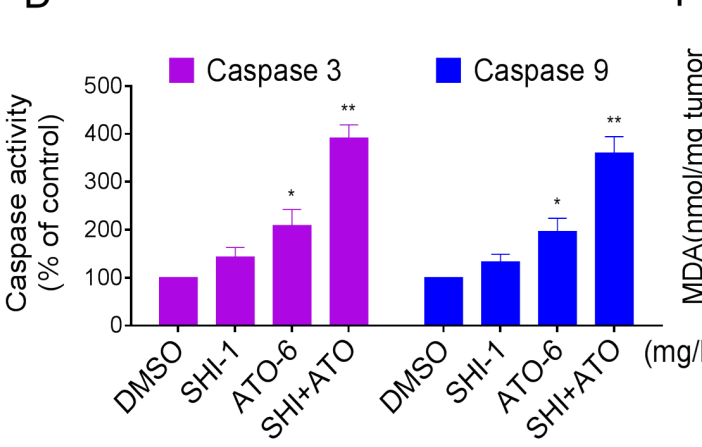

B

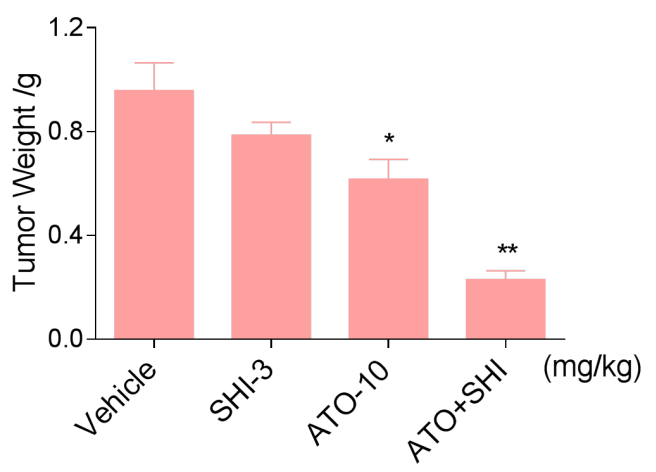

E

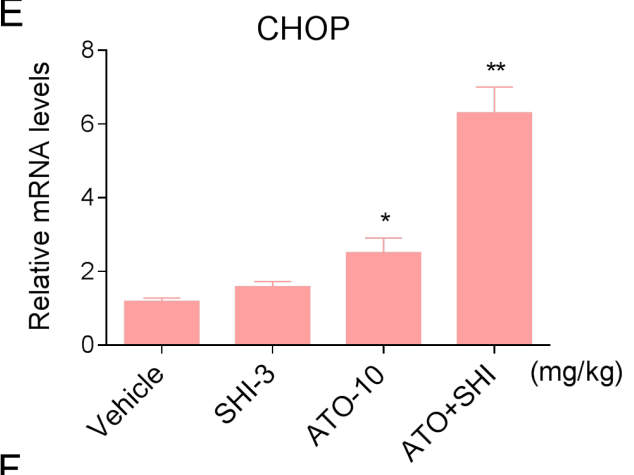

F

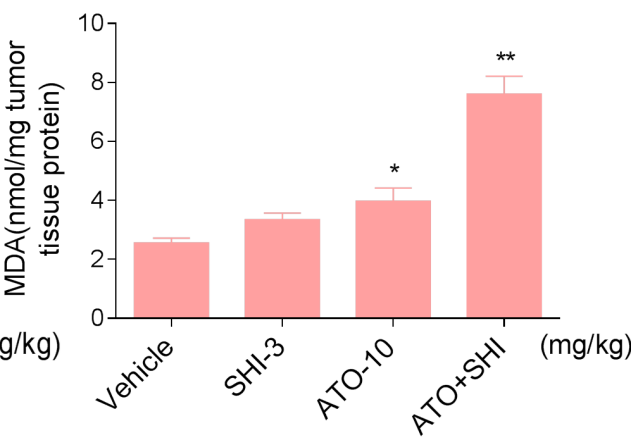

Figure 6: SHI increases the anticancer activity of ATO against HCC cells in vivo. Combined treatment inhibits tumor volume A. and tumor weight B. of HepG2 xenografts in nude mice, but do not affect body weight C. of mice. D. Combined treatment increased caspase 3 and caspase 9 activities in vivo. E. The levels of CHOP mRNA in the tumor tissues. F. The levels of MDA in the tumor tissues. $* \mathrm{P}<0.05$ versus control group, $* * \mathrm{P}<0.01$ versus control group. 
usually quite low [5]. Arsenic trioxide is an effective anticancer drug used in patients with relapsed acute promyelocytic leukemia, and is investigated for the treatment of unresectable HCC. However, the phase II study of ATO showed that single-agent ATO is not active against advanced HCC [9]. Therefore, the combination of ATO with a sensitizer that can enhance ATOmediated effects may be necessary for the treatment of HCC [31]. In the present study, we investigated the effect of SHI potentiates the cytotoxic effects of ATO in HCC cells. We found that combination of SHI and ATO is more effective than single agents in cancer cell lines. Furthermore, we found that SHI synergizes ATOmediated apoptosis by activating ROS-dependent ER stress pathway.

Over the years, apoptosis has received much attention in emerging as the major mechanism by which anti-tumor agents act to eliminate tumor cells. Apoptotic cells exhibit typical apoptotic features such as caspase activation, apoptotic body formation and DNA fragmentation [32]. We found that combination of SHI and ATO exerts a significant proapoptotic effect on HCC cells. Recent evidence showed that ATO induces apoptosis of cancer cells through activating ER stress pathway [23]. In the present study, we found that combined treatment with SHI and ATO significantly activated the ER stress pathway in HCC cells as evidenced by increased levels of p-EIF2 $\alpha$ and ATF4. Meanwhile, ER stress marker CHOP was significantly increased in HCC cells treated with SHI and ATO. Moreover, our findings demonstrated that blocking ER stress by 4-PBA significantly decreased combined treatment-induced apoptosis in HCC cells. Taken together, these findings suggested that ER stress was involved in combined treatment-induced apoptosis in HCC cells. Moreover, our findings indicated that targeting ER stress is a useful anti-cancer strategy and dissecting out these interactions in HCC cells will likely provide new targets for therapy.

Compared with normal cells, many types of cancer cell have increased levels of ROS. Therefore, it might be possible to selectively kill cancer cells by pharmacological ROS insults [14, 29]. In the previous studies, SHI exhibits potent anti-tumor activities via ROS-mediated apoptosis $[20,30]$. Our findings showed that SHI markedly increased ATO-induced apoptosis by promoting ROS production, and blockage of ROS production by NAC or GSH totally reversed the cell death induced by combined treatment with SHI and ATO in HepG2 cells. In addition, combined treatment-induced activation of ER stress and mitochondrial pathway were almost totally reversed by NAC, suggesting that ROS production is the upstream regulator. Previous study have shown that SHI interacts with thioredoxin reductase 1 to induce ROS in HL-60 cells [20]. Therefore, it is possible that SHI synergizes ATOmediated apoptosis in HCC cells by inhibiting thioredoxin reductase 1 activity. Elucidating these mechanisms is a pressing issue and a focus of future studies.

In conclusion, we investigated the synergistic anti-tumor effects of combined SHI and ATO in HCC cells and the potential underlying mechanisms. We found that SHI significantly enhanced ATO-induced HCC cells killing in vitro and in vivo, and demonstrated that combined treatment induces apoptotic cell death through increased production of ROS and activation of ER stress pathway. These results indicated that the combination of SHI and ATO possesses great potential as a promising strategy for the treatment of HCC. In addition, our findings indicated that ROS production and ER stress could be viewed as a potential strategy for the development of new anti-tumor agents.

\section{MATERIALS AND METHODS}

\section{Cell culture and reagents}

Arsenic trioxide (ATO), Shikonin (SHI), 4-phenylbutyric acid (4-PBA), N-acetylcysteine (NAC) and L-Glutathione reduced (GSH) were purchased from Sigma (St. Louis, MO). Human HCC cell lines HepG2, Hep3B, Huh7, and normal human liver cell line HL-7702 were purchased from the Institute of Biochemistry and Cell Biology, Chinese Academy of Sciences. The cells were cultured in RPMI-1640 medium containing 10\% Fetal Bovine Serum, 100 units/ml penicillin, and $100 \mu \mathrm{g} /$ $\mathrm{ml}$ streptomycin at $37^{\circ} \mathrm{C}$ in a humidified atmosphere with $5 \% \mathrm{CO}_{2}$. FITC Annexin V and Propidium Iodide (PI) were purchased from BD Pharmingen (Franklin Lakes, NJ). Antibodies against ATF4, p-EIF2 $\alpha$, EIF2 $\alpha$, and CHOP were purchased from Cell Signaling Technology (Danvers, MA). Antibodies against Bcl-2, Bax, caspase-3 p30/17, and GAPDH were purchased from Santa Cruz Biotechnology (Santa Cruz, CA).

\section{Cell viability assay}

Cells were seeded into 96-well plates at a density of $6 \times 10^{3}$ per well and allowed to grow overnight. ATO was dissolved in DMSO and diluted with 1640 medium to final concentrations of 2, 4, 6, 8, 10 and $12 \mu \mathrm{M}$. The cells were incubated with ATO or with a combination of ATO and SHI $(1 \mu \mathrm{M})$ for $24 \mathrm{~h}$ before the MTT assay.

\section{Cell apoptosis analysis}

Apoptosis was measured with FITC conjugated Annexin V and Propidium Iodide (PI) in binding buffer using a FACSCalibur flow cytometer (BD Biosciences, CA). Briefly, cells were washed twice with cold PBS, and then resuspended in $100 \mu \mathrm{L} 1 \times$ binding buffer. $5 \mu \mathrm{L}$ of annexin V-FITC and $5 \mu \mathrm{L}$ of PI were added. Thereafter, these cells were incubated for $15 \mathrm{~min}$ at room temperature in the dark, and then $400 \mu \mathrm{L}$ of binding buffer was added 
to each tube. The cells were analyzed by FACSCalibur flow cytometer.

\section{Western blot analysis}

Cells or tumor tissues were homogenized in protein lysate buffer, and debris was removed by centrifugation at $12,000 \mathrm{~g}$ for $12 \mathrm{~min}$ at $4^{\circ} \mathrm{C}$. The protein concentrations in all samples were determined by using the Bradford protein assay Kit (Bio-Rad, Hercules, CA). Protein samples were electrophoresed and then transferred to PVDF membrane. The membrane was blocked for $1 \mathrm{~h}$ at room temperature with fresh $5 \%$ nonfat milk in TBST, and then incubated with primary antibody overnight at $4{ }^{\circ} \mathrm{C}$ and secondary antibody for one hour at room temperature. The immunoreactive bands were visualized by using ECL Kit (Bio-Rad, Hercules, CA). The density of the immunoreactive bands was analyzed using Image $\mathrm{J}$ computer software.

\section{Quantitative RT-PCR}

Cells were homogenized in TRIzol reagent (Invitrogen, Carlsbad, CA) for extraction of RNA according to each manufacturer's protocol. Both reverse transcription and quantitative PCR were carried out using a two-step M-MLV Platinum SYBR Green qPCR SuperMix-UDG Kit (Invitrogen, Carlsbad, CA). Eppendorf Mastercycler eprealplex detection system (Eppendorf, Hamburg, Germany) was used for q-PCR analysis. The following gene-specific primer pairs were used: CHOP: (F) 5'-atggcagctgagtcattgcetttc-3', (R) 5'-agaagcagggtcaagagtggtgaa-3'. $\beta$-actin: (F) 5'-ttcctgggcatg gagtcct-3', (R) 5'-aggaggagcaatgatcttgatc-3'. Gene expressions were analyzed with the comparative threshold cycle $(\mathrm{Ct})$ method after normalizing to the housekeeping gene $\beta$-actin.

\section{Measurement of reactive oxygen species}

Cellular ROS levels were measured by flow cytometer. Briefly, cells were plated on $60-\mathrm{mm}$ dishes and allowed to grow overnight. After treatment, cells were stained with $10 \mu \mathrm{M}$ DCFH-DA (Beyotime Biotech, China) at $37^{\circ} \mathrm{C}$ for $30 \mathrm{~min}$. Cells were collected and the fluorescence was analyzed using a FACSCalibur flow cytometer.

\section{Determination of caspase 3/9 activity}

Caspase 3/9 activity in cell lysates was determined using a Caspase 3/9 activity Kit (Beyotime Biotech, China) according to the manufacturer's protocol. The caspase 3/9 activity was normalized by the protein concentration of the corresponding cell lysate and expressed as percentage of treated cells to that of control.

\section{In vivo antitumor study}

All surgical procedures and care administered to the animals were in accordance with institutional animal ethic guidelines. Animals were housed at a constant room temperature with a $12 \mathrm{~h}$ light $/ 12 \mathrm{~h}$ dark cycle and fed a standard rodent diet and water. Tumors were established by subcutaneous injection of $5 \times 10^{6} \mathrm{HepG} 2$ tumor cells into the flanks of mice. When tumors reached a volume of about $100 \mathrm{~mm}^{3}$, the mice were randomly assigned to 4 groups (each group had seven mice): control, SHI, ATO, ATO + SHI. Mice were treated by intraperitoneal (i.p.) injection of $10 \mathrm{mg} / \mathrm{kg}$ ATO once per day, or by i.p. injection of $3 \mathrm{mg} / \mathrm{kg}$ SHI once per day, or with a combination of ATO and SHI according to the same schedules. The tumor volumes were determined by measuring length (l) and width (w) and calculating volume $\left(\mathrm{V}=0.5 \times 1 \times \mathrm{w}^{2}\right)$ at the indicated time points. At the end of treatment, the animals were sacrificed, and the tumors were removed and weighed.

\section{Statistical analysis}

All experiments were assayed in triplicate $(\mathrm{n}=$ 3). Data are expressed as means \pm SEM. All statistical analyses were performed using GraphPad Pro. Prism 5.0. Student's t-test and two-way ANOVA were employed to analyze the differences between sets of data. A $p$ value $<0.05$ was considered statistically significant.

\section{ACKNOWLEDGMENTS}

The work was supported by National Natural Science Foundation of China (81573657) and the major social development projects of Zhejiang major science and technology projects (2013C03010).

\section{CONFLICTS OF INTEREST}

The authors disclose no potential conflicts of interest.

\section{REFERENCES}

1. El-Serag HB. Hepatocellular carcinoma. The New England journal of medicine. 2011; 365:1118-1127.

2. Hollebecque A, Malka D, Ferte C, Ducreux M, Boige V. Systemic treatment of advanced hepatocellular carcinoma: from disillusions to new horizons. Eur J Cancer. 2015; 51:327-339.

3. Worns MA, Galle PR. HCC therapies--lessons learned. Nature reviews Gastroenterology \& hepatology. 2014; $11: 447-452$.

4. El-Serag HB, Marrero JA, Rudolph L, Reddy KR. Diagnosis and treatment of hepatocellular carcinoma. Gastroenterology. 2008; 134:1752-1763. 
5. Llovet JM, Ricci S, Mazzaferro V, Hilgard P, Gane E, Blanc JF, de Oliveira AC, Santoro A, Raoul JL, Forner A, Schwartz M, Porta C, Zeuzem S, et al. Sorafenib in advanced hepatocellular carcinoma. The New England journal of medicine. 2008; 359:378-390.

6. Lo-Coco F, Avvisati G, Vignetti M, Thiede C, Orlando SM, Iacobelli S, Ferrara F, Fazi P, Cicconi L, Di Bona E, Specchia G, Sica S, Divona M, et al. Retinoic acid and arsenic trioxide for acute promyelocytic leukemia. The New England journal of medicine. 2013; 369:111-121.

7. Zhou W, Cheng L, Shi Y, Ke SQ, Huang Z, Fang X, Chu CW, Xie Q, Bian XW, Rich JN, Bao S. Arsenic trioxide disrupts glioma stem cells via promoting PML degradation to inhibit tumor growth. Oncotarget. 2015; 6:37300-37315. doi: 10.18632/oncotarget.5836.

8. Liu B, Pan S, Dong X, Qiao H, Jiang H, Krissansen GW, Sun X. Opposing effects of arsenic trioxide on hepatocellular carcinomas in mice. Cancer science. 2006; 97:675-681.

9. Lin CC, Hsu C, Hsu CH, Hsu WL, Cheng AL, Yang $\mathrm{CH}$. Arsenic trioxide in patients with hepatocellular carcinoma: a phase II trial. Investigational new drugs. 2007; 25:77-84.

10. Podrazil M, Horvath R, Becht E, Rozkova D, Bilkova P, Sochorova K, Hromadkova H, Kayserova J, Vavrova K, Lastovicka J, Vrabcova P, Kubackova K, Gasova $\mathrm{Z}$, et al. Phase $\mathrm{I} / \mathrm{II}$ clinical trial of dendritic-cell based immunotherapy (DCVAC/PCa) combined with chemotherapy in patients with metastatic, castrationresistant prostate cancer. Oncotarget. 2015; 6:18192-18205. doi: 10.18632/oncotarget.4145.

11. Vanden Borre P, Gunda V, McFadden DG, Sadow PM, Varmeh S, Bernasconi M, Parangi S. Combined BRAF(V600E)- and SRC-inhibition induces apoptosis, evokes an immune response and reduces tumor growth in an immunocompetent orthotopic mouse model of anaplastic thyroid cancer. Oncotarget. 2014; 5:3996-4010. doi: 10.18632/oncotarget.2130.

12. Trachootham D, Alexandre J, Huang P. Targeting cancer cells by ROS-mediated mechanisms: a radical therapeutic approach? Nature reviews Drug discovery. 2009; 8:579-591.

13. Gorrini C, Harris IS, Mak TW. Modulation of oxidative stress as an anticancer strategy. Nature reviews Drug discovery. 2013; 12:931-947.

14. Raj L, Ide T, Gurkar AU, Foley M, Schenone M, Li X, Tolliday NJ, Golub TR, Carr SA, Shamji AF, Stern AM, Mandinova A, Schreiber SL, Lee SW. Selective killing of cancer cells by a small molecule targeting the stress response to ROS. Nature. 2011; 475:231-234.

15. Hui KF, Yeung PL, Chiang AK. Induction of MAPK- and ROS-dependent autophagy and apoptosis in gastric carcinoma by combination of romidepsin and bortezomib. Oncotarget. 2016; 7:4454-4467. doi: 10.18632/oncotarget.6601.
16. Gu S, Chen C, Jiang X, Zhang Z. ROS-mediated endoplasmic reticulum stress and mitochondrial dysfunction underlie apoptosis induced by resveratrol and arsenic trioxide in A549 cells. Chemico-biological interactions. 2016; 245:100-109.

17. Kumar S, Yedjou CG, Tchounwou PB. Arsenic trioxide induces oxidative stress, DNA damage, and mitochondrial pathway of apoptosis in human leukemia (HL-60) cells. Journal of experimental \& clinical cancer research. 2014; $33: 42$.

18. Zhao Q, Assimopoulou AN, Klauck SM, Damianakos H, Chinou I, Kretschmer N, Rios JL, Papageorgiou VP, Bauer R, Efferth T. Inhibition of c-MYC with involvement of ERK/JNK/MAPK and AKT pathways as a novel mechanism for shikonin and its derivatives in killing leukemia cells. Oncotarget. 2015; 6:38934-38951. doi: 10.18632/oncotarget.5380.

19. Singh F, Gao D, Lebwohl MG, Wei H. Shikonin modulates cell proliferation by inhibiting epidermal growth factor receptor signaling in human epidermoid carcinoma cells. Cancer letters. 2003; 200:115-121.

20. Duan D, Zhang B, Yao J, Liu Y, Fang J. Shikonin targets cytosolic thioredoxin reductase to induce ROS-mediated apoptosis in human promyelocytic leukemia HL-60 cells. Free radical biology \& medicine. 2014; 70:182-193.

21. Ahn J, Won M, Choi JH, Kim YS, Jung CR, Im DS, Kyun ML, Lee K, Song KB, Chung KS. Reactive oxygen speciesmediated activation of the Akt/ASK1/p38 signaling cascade and p21(Cip1) downregulation are required for shikonininduced apoptosis. Apoptosis. 2013; 18:870-881.

22. Gong K, Li W. Shikonin, a Chinese plant-derived naphthoquinone, induces apoptosis in hepatocellular carcinoma cells through reactive oxygen species: A potential new treatment for hepatocellular carcinoma. Free radical biology \& medicine. 2011; 51:2259-2271.

23. Chiu HW, Tseng YC, Hsu YH, Lin YF, Foo NP, Guo HR, Wang YJ. Arsenic trioxide induces programmed cell death through stimulation of ER stress and inhibition of the ubiquitin-proteasome system in human sarcoma cells. Cancer letters. 2015; 356:762-772.

24. Zhang H, Duncan G, Wang L, Liu P, Cui H, Reddan JR, Yang BF, Wormstone IM. Arsenic trioxide initiates ER stress responses, perturbs calcium signalling and promotes apoptosis in human lens epithelial cells. Experimental eye research. 2007; 85:825-835.

25. Gara RK, Srivastava VK, Duggal S, Bagga JK, Bhatt M, Sanyal S, Mishra DP. Shikonin selectively induces apoptosis in human prostate cancer cells through the endoplasmic reticulum stress and mitochondrial apoptotic pathway. Journal of biomedical science. 2015; 22:26.

26. Chouchani ET, Kazak L, Jedrychowski MP, Lu GZ, Erickson BK, Szpyt J, Pierce KA, Laznik-Bogoslavski D, Vetrivelan R, Clish CB, Robinson AJ, Gygi SP, Spiegelman BM. Mitochondrial ROS regulate thermogenic energy 
expenditure and sulfenylation of UCP1. Nature. 2016; 532:112-116.

27. Poillet-Perez L, Despouy G, Delage-Mourroux R, BoyerGuittaut M. Interplay between ROS and autophagy in cancer cells, from tumor initiation to cancer therapy. Redox biology. 2015; 4:184-192.

28. Chen Y, Liu JM, Xiong XX, Qiu XY, Pan F, Liu D, Lan SJ, Jin S, Yu SB, Chen XQ. Piperlongumine selectively kills hepatocellular carcinoma cells and preferentially inhibits their invasion via ROS-ER-MAPKs-CHOP. Oncotarget. 2015; 6:6406-6421. doi: 10.18632/oncotarget.3444.

29. Yu X, Deng Q, Li W, Xiao L, Luo X, Liu X, Yang L, Peng S, Ding Z, Feng T, Zhou J, Fan J, Bode AM, Dong Z, Liu J, Cao Y. Neoalbaconol induces cell death through necroptosis by regulating RIPK-dependent autocrine TNFalpha and
ROS production. Oncotarget. 2015; 6:1995-2008. doi: 10.18632/oncotarget.3038.

30. Mao X, Yu CR, Li WH, Li WX. Induction of apoptosis by shikonin through a ROS/JNK-mediated process in Bcr/Ablpositive chronic myelogenous leukemia (CML) cells. Cell research. 2008; 18:879-888.

31. Khurana A, Roy D, Kalogera E, Mondal S, Wen X, He X, Dowdy S, Shridhar V. Quinacrine promotes autophagic cell death and chemosensitivity in ovarian cancer and attenuates tumor growth. Oncotarget. 2015; 6:36354-36369. doi: 10.18632/oncotarget.5632.

32. Okada H, Mak TW. Pathways of apoptotic and nonapoptotic death in tumour cells. Nature reviews Cancer. 2004; 4:592-603. 